\title{
Vapor phase SERS sensor based on mesoporous silica decorated with silver nanoparticles
}

\author{
A. S. Sarycheva ${ }^{1}$, A. A. Semenova ${ }^{2}$, E. A. Goodilin ${ }^{2,3, *}$ \\ ${ }^{1}$ Department of Materials Science and Engineering and A. J. Drexel Nanomaterials Institute, \\ Drexel University, Philadelphia, PA 19104, USA \\ ${ }^{2}$ Lomonosov Moscow State University, Faculty of Materials Science, Lenin Hills, Moscow, 119991, Russia \\ ${ }^{3}$ Kurnakov Institute of General and Inorganic Chemistry of the Russian Academy of Sciences Sciences, \\ Moscow, 119991, Russia \\ *goodilin@yandex.ru
}

PACS 61.46.Hk, 62.23.St, 68.35.bd

DOI 10.17586/2220-8054-2017-8-5-579-585

\begin{abstract}
For the first time, nanocomposite colloidosomes of mesoporous silica microspheres decorated with silver nanoparticles have been applied as a highly sensitive vapor phase optical sensors utilizing a semiquantitative analysis of surface-enhanced Raman spectra (SERS). The material was prepared using soft chemistry approaches and benefits from the intrinsic properties of both components: the mesoporous structure of the silica microspheres allows for capillary condensation of target analytes while the silver nanoparticles favor the great enhancement of Raman fingerprints of thus trapped and preconcentrated analytes. This approach seems to be highly promising for the further development of express gas phase sensors for heterocyclic or polycyclic aromatic hydrocarbon pollutants.
\end{abstract}

Keywords: SERS, silver, nanostructures, nanoparticles, preparation methods, background.

Received: 1 September 2017

Revised: 22 September 2017

\section{Introduction}

Recently, the rapid development of Surface-enhanced Raman spectroscopy (SERS) is supported greatly by its obvious advantageous features, such as remarkable sensitivity, label-free techniques of use, robust and express practical application routes $[1,2]$. At the same time, ordinary materials for SERS with ecological, pharmaceutical, biological and medical applications are commonly designed for the analysis of liquid analytes [1-10] while a limited number of actual research efforts has focused on vapor phase detection [11-29]. The latter challenge evidently widens the promising application area of SERS and therefore requires novel approaches for the design of SERS-active materials.

At present, most of the research advantages for SERS in the analysis of gaseous and vapor phases are based on simple chemical sorption of analytes on a highly developed silver or gold surface if they have high affinity to plasmonic nanoparticles being, for example, thiol derivatives or other similar molecules $[11,13,14,19,20,25,27,29]$. Accordingly, the sensitivity of the analysis becomes even higher if porous silver nanocubes [12], polymer coatings [15], anisotropic nanoparticle aggregates [16,24], metal - organic frameworks [21,26], multihole capillaries [23], graphene oxide surface [28], free - surface microfluidic chips [18], electrodynamic precipitation [17] are applied.

In this paper, nanocomposite colloidosomes of mesoporous silica microspheres [30,31] decorated with silver nanoparticles are applied for the first time as a highly sensitive vapor phase optical sensor utilizing a semiquantitative SERS analysis. We suggest that the material prepared using soft chemistry approaches benefits from the intrinsic properties of both the precursors: the silica microspheres' mesoporous structure allows for capillary condensation of target analytes; the silver nanoparticles favor the significant enhancement of Raman fingerprints of trapped and preconcentrated analytes.

\section{Experimental}

Highly pure water (Milli-Q, Millipore), tetraethylorthosilicate (TEOS), cetyl trimethylammonium bromide (CTAB), silver $(\mathrm{I})$ nitrate $\left(\mathrm{AgNO}_{3}\right)$, sodium citrate, sodium hydroxide $(\mathrm{NaOH})$, ethyl alcohol, methanol, hydrochloric acid $(\mathrm{HCl})$, aqueous ammonia solution, polyvinylpyrrolidone (PVP), sodium borohydride $\left(\mathrm{NaBH}_{4}\right)$, ascorbic acid (Aldrich) were used as reagents.

Silica microspheres were synthesized by the Stöber method [30,31] using the ammonia-catalyzed hydrolysis of TEOS at $400{ }^{\circ} \mathrm{C}$ for $5-10 \mathrm{~h}$ with the molar ratio of the components $\mathrm{C}_{2} \mathrm{H}_{5} \mathrm{OH}: \mathrm{NH}_{3}:$ TEOS : $\mathrm{H}_{2} \mathrm{O}=$ 
$63: 13: 4: 1$. Typically, to prepare $500 \mathrm{ml}$ of suspension, solution of $367 \mathrm{ml}$ of ethyl alcohol, $101 \mathrm{ml}$ of $25 \%$ ammonia and $10 \mathrm{ml}$ of water was heated up to $40{ }^{\circ} \mathrm{C}$ with magnetic stirring $(400 \mathrm{rpm}$ ) followed by addition of 9 $\mathrm{ml}$ of TEOS and maintaining the mixture overnight (12 hrs) to complete hydrolysis and silica microsphere growth. The as-prepared suspension was repeatedly centrifuged $(6000 \mathrm{rpm}, 15 \mathrm{~min})$ and washed (redispersed) with water to remove residual reagents. Finally, the predried sample was slowly heated up to $550{ }^{\circ} \mathrm{C}$ in air $\left(1{ }^{\circ} \mathrm{C} / \mathrm{min}\right)$ and held for 5 hrs followed by furnace cooling. Similarly, MCM- 41 mesoporous silica microspheres were prepared in the presence of CTAB as a structure-directing surfactant [30,31]. Typically, $7.5 \mathrm{~g}$ of CTAB was mixed with 150 $\mathrm{ml}$ of pure water and heated up to $40{ }^{\circ} \mathrm{C}$ under vigorous stirring. The resulting soap-like semitransparent liquid was kept at room temperature followed by addition of $47 \mathrm{ml}$ of aqueous ammonia $(25-27 \%)$ and $77 \mathrm{ml}$ of ethyl alcohol under stirring for $15 \mathrm{~min}$. After that, $15 \mathrm{ml}$ of dry TEOS was added under stirring and the mixture was stirred for $20 \mathrm{hrs}$. The resulting suspension was filtered, washed with pure water and methanol followed by drying for $20 \mathrm{hrs}$ at $90{ }^{\circ} \mathrm{C}$. An additional structure saving preparation stage of surfactant extraction at gentle temperatures was applied to enhance absorption of molecules in a gas state. In the most of cases $1 \mathrm{~g}$ of mesoporous silica microspheres was placed into $107 \mathrm{ml}$ of methanol and $2.8 \mathrm{ml}$ of $1 \mathrm{M} \mathrm{HCl}$. The mixture was refluxed at $60{ }^{\circ} \mathrm{C}$ for $24 \mathrm{hrs}$ followed by washing with ethanol and drying at $90{ }^{\circ} \mathrm{C}$ in air.

Soft chemistry decoration of silica microspheres was performed using their reaction with hot solutions of diamminesilver (I) hydroxide. To obtain diamminesilver (I) hydroxide, $0.1 \mathrm{M}$ aqueous sodium hydroxide was added dropwise to a freshly prepared $0.01 \mathrm{M}$ aqueous silver nitrate solution until complete precipitation of a blackbrown silver (I) oxide. Thus, the prepared silver (I) oxide was thoroughly washed with water and dissolved in a two-fold molar excess of a $10 \%$ aqueous ammonia solution, resulting in the formation of $0.01 \mathrm{M}$ diamminesilver (I) hydroxide. The obtained transparent complex solution was filtered through Millex-LCR syringe driven filter units (Millipore, $0.45 \mathrm{~mm}$ pores). After that, typically $0.3 \mathrm{~g}$ of Stöber silica was redispersed in $10 \mathrm{ml}$ of pure water by ultrasound treatment for $30 \mathrm{~min}$. Such a suspension was mixed in the ratio of 1:1 with the preliminary prepared diamminesilver (I) hydroxide solution, heated at $95-97{ }^{\circ} \mathrm{C}$, and held for $0.5-2.0 \mathrm{hrs}$, filtered, repeatedly washed with pure water and dried to prepare yellow-brown powder of mesoporous silica colloidosomes decorated with silver nanoparticles. In a number of cases, silver nanoparticles sizes were increased by their regrowth by additional reduction of silver nitrate with ascorbic acid in the presence of an aqueous suspension of the colloidosomes.

The obtained colloidosomes were characterized by scanning electron microscopy (Carl Zeiss NVision 40) at $0.5-5 \mathrm{kV}$ accelerating voltage. The materials dried on alumina plates were examined using Rigaku D/MAX 2500 (Japan) with a rotating copper anode $\left(\mathrm{CuK}_{\text {alpha }}\right.$ irradiation, $5-80^{\circ} 2$ Theta range, $0.02^{\circ}$ step). Diffraction maxima were indexed using the PDF-2 database. UV-vis absorption spectra were recorded using the UV-vis spectrophotometer Lambda 35 (Perkin-Elmer) with an attached diffuse reflectance accessory. Size distribution and zeta potentials measurements were performed using Malvern Zetasizer Nano ZS. Thermal analysis assisted by evaluation of the exhausted products composition was utilized using the thermal analyzer STA 409 PC Luxx with an attached gas analyzer QMS $403 \mathrm{D}$ Aëolos (NETZSCH) at a heating rate of $3{ }^{\circ} \mathrm{C} / \mathrm{min}$ in air in the temperature range of $30-900{ }^{\circ} \mathrm{C}$. BET and $\mathrm{BJH}$ analysis were done using the Quantachrome NOVA 4200e setup.

Raman and SERS experiments were performed using the InVia Raman microscope (Renishaw, UK) equipped with a $20 \mathrm{~mW} 514 \mathrm{~nm}$ argon laser and power neutral density filters. All the spectra were collected using $\times 5$ objective, NA 0.15 and $10-30 \mathrm{~s}$ of acquisition time. A silicon wafer was used for calibration.

\section{Results and discussion}

Pyridine is a classical model compound for SERS measurements and it is selected in the present paper as a primary analyte because of several important reasons. First of all, this was the first compound detected by SERS in 1973 after its adsorption onto electrochemically deposited silver [32], also, it is known that affinity of pyridine to silver is high enough and the surface layer seems to be strong due to chemical bonding. The compound demonstrates high delocalization of $\pi$ electrons and this effect, as well known, increases much SERS enhancement of such molecules. The pyridine normal modes with the largest Raman intensity are 983 and $1026 \mathrm{~cm}^{-1}$ corresponding to ring breathing; the mode at $598 \mathrm{~cm}^{-1}$ is a ring deformation mode; ring stretching modes appear at 1208, 1472 and $1583 \mathrm{~cm}^{-1}$. The most intense peak at $1001 \mathrm{~cm}^{-1}$ appear usually in SERS spectra because of a shift by about $20 \mathrm{~cm}^{-1}$ due to a chemical interaction with silver surface. Therefore this particular mode could be selected as a reference peak for the estimation of the amount of pyridine. In addition, the boiling point of pyridine at $116{ }^{\circ} \mathrm{C}$ favors capillary adsorption. Finally, pyridine is a typical heterocyclic component of crude oil and its content is important to analyze to determine oil quality.

The samples prepared for the SERS analysis of pyridine represent silver - silica nanocomposites with a highly porous structure (Fig. 1). The nucleus of silver clusters are distributed homogeneously after the reaction of silica microspheres with hot diamminesilver (I) solution (Fig. 1(a,b)). Larger silver nanoparticles formed onto microporous 
silver colloidosomes are attached to the surface (Fig. 1(c, d)). The overall silver content in the nanocomposites is dependent on preparation parameters in a complex way (Fig. 1(e)), the latter is connected with two competing processes - silver cluster deposition and superficial etching with alkali solution of diamminesilver (I) hydroxide. It seems that silver concentrations of $10 \mathrm{mM}$ and reacting time of $20-60 \mathrm{~min}$ are suitable conditions for the formation of the nanocomposite. The most preferable treatment procedure of the silica microspheres with hot diamminsilver (I) hydroxide includes the shortest $(20 \mathrm{~min})$ reaction time (Fig. 1). As a result, it minimizes silica dissolution and its surface erosion while generation of uniformly distributed superficial silver nanoparticle of 3 $5 \mathrm{~nm}$ remains effective. This is connected with remaining ethoxy groups of silica participating in silver reduction and nanoparticles attachment to the microspheres. The measured surface area of the mesoporous samples exceeds $1100 \mathrm{~cm}^{2} / \mathrm{g}$ and becomes by about $10 \%$ higher after the surfactant extraction procedure compared to traditional annealing and therefore extraction seems to be a predominant technique for creation of open pores (Fig. 1(f)). The nanocomposite demonstrates plasmon resonance modes around $400 \mathrm{~nm}$ typical of isotropic silver nanoparticles (Fig. 1(g)) which is also confirmed either by an XRD analysis (Fig. 1(h)) demonstrating all clear diffraction peaks of cubic metallic silver or probe SERS measurements of a model Rhodamine 6G dye showing most intense Raman modes at low concentrations (Fig. 1(i)). The complex investigation of the colloidosomes thus confirms (Fig. 1) that they represent a SERS-active nanocomposite material with a high surface area.

Such a material is a good candidate for SERS investigation of gaseous phases and it was used then to detect pyridine in the vapor phase at different partial pressures of the analyte to determine a wide range of volumetric concentrations in a carrier gas (heptane) having a low Raman intensities compared to pyridine. Both the organic components, pyridine or heptane, could be absorbed by the colloidosomes but this process seems to be more preferable for pyridine due to its chemical nature while heptane plays the role of an inert diluting component (Fig. 2).

Figure 2 shows typical intensities of a narrow-selected region of raw measurements of the most intense enhanced Raman modes of pyridine, absorbed by the porous structure of the colloidosomes after exposure of the SERS-active material in a gaseous mixture of pyridine and heptane with different partial pressures (volumetric content) of pyridine. It is evident that there is a distinct correlation between the content of pyridine in vapor and the spectral features of this compound measured using plasmonic nanoparticles of silver decorating mesoporous silica. The inset of Fig. 2 demonstrates an almost linear dependence between the SERS intensities and concentrations under optimal measurement conditions at a low concentration end. The detection limit of this compound comprises a better mark that $10-100 \mathrm{ppm}$. It should be noted that this sensitivity level is found for a two-component mixture with heptane which seems to be generally similar with a gaseous atmosphere over some oil reforming products. Probably, a vapor over car fuel will contain, at least, several more components including benzene which would also be absorbed by the colloidosomes and will reveal itself in SERS spectra. However the discussed system is a simpler model example showing a possibility of such an analysis near pyridine safety concentration threshold using the prepared type of SERS materials.

Mesoporous channels, being definitely present in the colloidosomes (Fig. 1), favor capillary condensation of both the organic components of the vapor phase (Fig. 3). Boiling points of pyridine at $116{ }^{\circ} \mathrm{C}$ and heptane at $98^{\circ} \mathrm{C}$ are very close and therefore it would be expected that they both will be equally absorbed however a distinct weight loss of about $4 \%$ at $130-160{ }^{\circ} \mathrm{C}$ accompanied by desorption of constituents with a molecular mass of 79 proves that it is mostly pyridine that physically absorbed and releases slightly above its boiling temperature. Another event is observed at $300-450{ }^{\circ} \mathrm{C}$ and this wide temperature range most probably corresponds to oxidation of the residual organic with the formation of $\mathrm{N}_{2}, \mathrm{H}_{2} \mathrm{O}, \mathrm{NH}_{3}, \mathrm{CO}_{2}, \mathrm{NO}_{2}$ and $\mathrm{NO}$. It is not found also that bromine could be evolved from the pores meaning that the extraction procedure was effective and CTAB, which might be a generator of heavy bromine-containing vaporized compounds, is not really present in the colloidosomes. It is also important to note that it is known that thermal oxidation of pyridine studied using a matrix-isolation infrared spectroscopy gives almost similar products [33] as we found in our experiments: carbon monoxide or nitrogen, carbon dioxide, nitric oxides and some others evidencing for oxidation of a pseudoaromatic ring containing a nitrogen atom. In such a case, the molecules found are related to burning to either pyridine or heptane capillary condensed in the mesopores. This is the only phenomenon in this system which can keep organic molecules inside the colloidosomes at temperature as high as $400{ }^{\circ} \mathrm{C}$. 

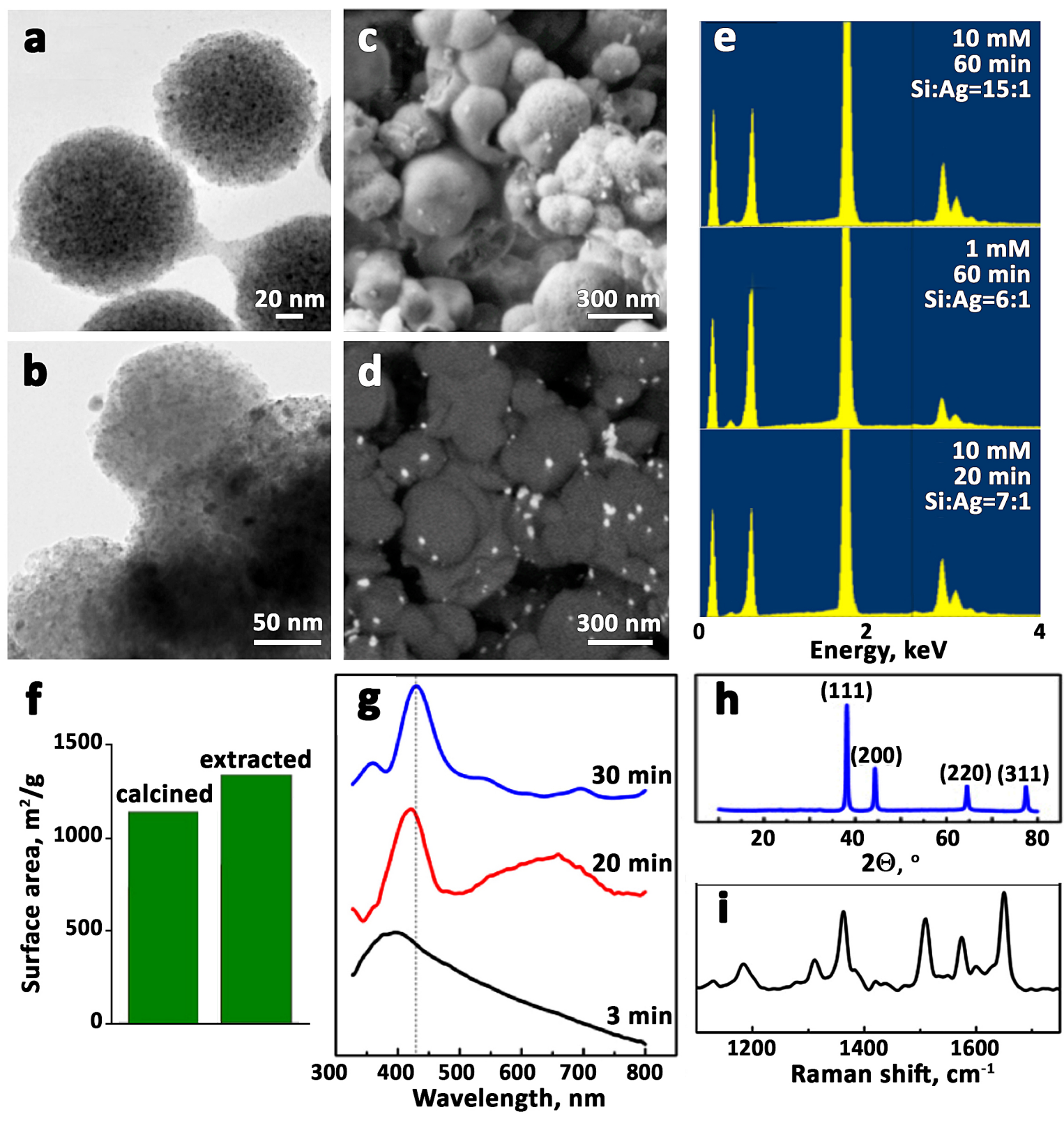

FIG. 1. Colloidosomal material characterization. (a, b) TEM images of silica microspheres decorated with silver nanoparticles; (c, d) SEM micrographs of mesoporous silica in SE and BSE modes demonstrating silver nanoparticle aggregates; (e) EDX analysis with respect to the Si:Ag ratio for the samples with a different preparation history; (f) comparison of porous structure of silica after surfactant thermal destruction or extraction; (g) plasmon resonance modes of the samples with different reaction time of pristine silica with a hot solution of diamminesilver (I) hydroxide; (h) typical XRD pattern of the composite; (i) SERS spectrum of a model dye Rhodamine $6 \mathrm{G}$ at $10^{-8} \mathrm{M}$ concentration 


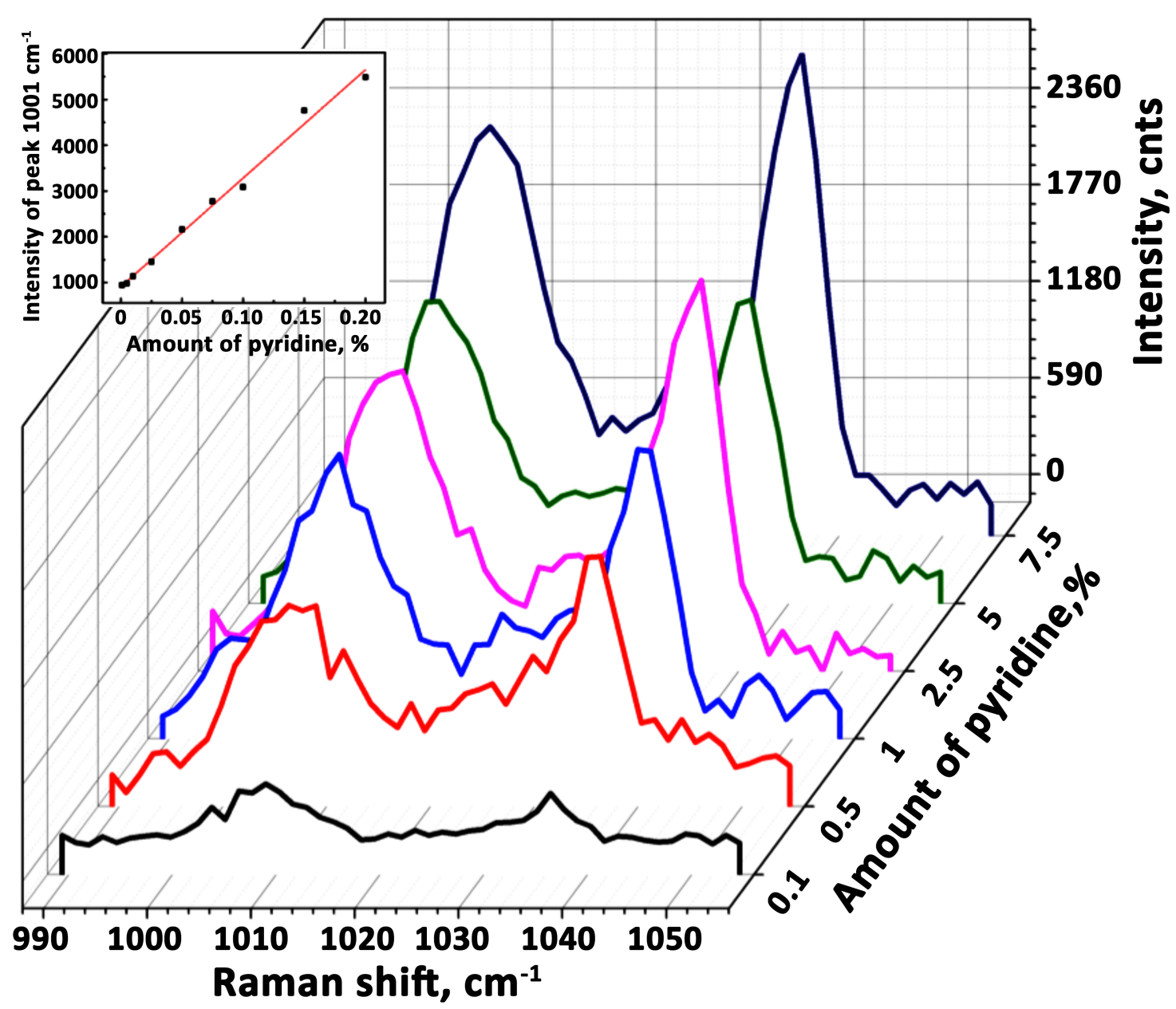

FIG. 2. Experimental data on SERS enhancement of typical spectral modes of pyridine absorbed by silver decorated colloidosomes of mesoporous silica exposed for $0.5-1 \mathrm{~min}$ in a mixed vapor phase of pyridine and heptane when varying volumetric (molar) content of the target analyte. The inset shows a nearly linear approximation of the dependence of the intensity of the $1001 \mathrm{~cm}^{-1}$ peak on concentration

\section{Conclusions}

Thus nanocomposite colloidosomes of mesoporous silica microspheres decorated with silver nanoparticles have been successfully applied as highly sensitive vapor phase optical sensors utilizing a semiquantitative analysis of surface-enhanced Raman spectra. The material was prepared using soft chemistry approaches and benefits from the intrinsic properties for both of the components: the mesoporous structure of the silica microspheres allows for capillary condensation of target analytes while the silver nanoparticles favor the great enhancement of Raman fingerprints of thus trapped and preconcentrated analytes at low concentrations in gaseous phase. This approach would be recommended as a further development of universal gas phase sensors for volatile organic compounds. 


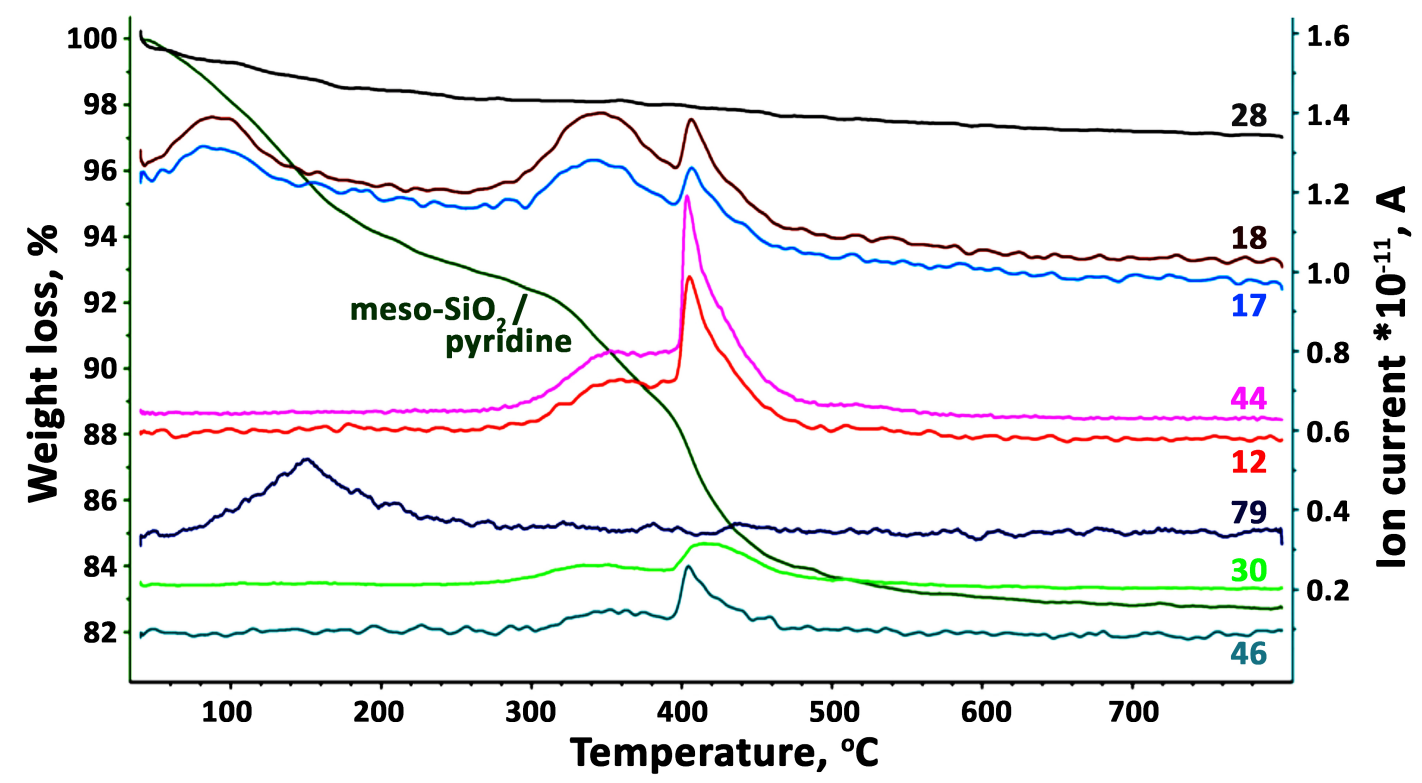

FIG. 3. Experimental data on a simultaneous TGA and mass-spectrometry analysis of outlet gases for silver-decorated colloidosomes of mesoporous silica with pyridine adsorbed upon their exposure in a vapor phase in SERS experiments. The TG curve is marked with the "meso$\mathrm{SiO}_{2}$ /pyridine" label. Other curves show the amounts of different molecular fragments released from the colloidosomes, presumably, 28 means $\mathrm{CO}$ or $\mathrm{N}_{2}, 18-\mathrm{H}_{2} \mathrm{O}, 17-\mathrm{NH}_{3}, 44-\mathrm{CO}_{2}, 79-$ pyridine, $46-\mathrm{NO}_{2}, 30-\mathrm{NO}$. Molecular bromine with a molecular mass of 158 and heptane itself with a molecular mass 100 are not detected

\section{Acknowledgements}

The work is supported by the Russian Science Foundation (grant 14-13-00871). Authors thank V. K. Ivanov and A. E. Baranchikov for SEM measurements and fruitful discussions.

\section{References}

[1] Yuan Y., Panwar N., et al. SERS-based ultrasensitive sensing platform: An insight into design and practical applications. Coord. Chem. Rev., 2017, 337, P. 1-33.

[2] Sharma B., Frontiera R.R., et al. SERS: Materials, applications, and the future. Mater. Today, 2012, 15 (1-2), P. $16-25$.

[3] Semenova A.A., Semenov A.P., et al. Nanostructured silver materials for noninvasive medical diagnostics by surface-enhanced Raman spectroscopy (focus article). Mendeleev Commun., 2016, 26 (3), P. 177-186.

[4] Brazhe N.A., Evlyukhin A.B., et al. Probing cytochrome c in living mitochondria with surface-enhanced Raman spectroscopy. Sci. Rep., 2015, 5, 13793.

[5] Sarycheva A.S., Brazhe N.A., et al. New nanocomposites for SERS studies of living cells and mitochondria. J. Mater. Chem. B, 2016, 3 (4), P. 539-546.

[6] Sarycheva A.S., Ivanov V.K., et al. Microbead silica decorated with polyhedral silver nanoparticles as a versatile component of sacrificial gel films for SERS applications. RSC advances, 2015, 5 (110), P. 90335-90342.

[7] Semenova A.A., Ivanov V.K., Savilov S.V., Goodilin E.A. Unusual silver nanostructures prepared by aerosol spray pyrolysis. Cryst. Eng. Comm., 2013, 15 (39), P. 7863-7871.

[8] Semenova A.A., Brazhe N.A., et al. A new route of SERS analysis of intact erythrocytes using polydisperse silver nanoplatelets on biocompatible scaffolds. RSC advances, 2016, 6 (88), P. 85156-85164.

[9] Semenova A.A., Goodilin E.A., et al. Planar SERS nanostructures with stochastic silver ring morphology for biosensor chips. J. Mater. Chem., 2012, 22 (47), P. 24530-24544.

[10] Semenova A.A., Semenova I.A., et al. Revisiting preparation routes of SERS materials. Nanosystems: Physics, Chemistry, Mathematics, 2017, 8 (5), P. 670-676.

[11] Hill W., Wehling B., Klockow D. Analysis of odorous vapor exhaled from rubber by surface-enhanced Raman scattering. Appl. Spectrosc., 1999, 53 (5), P. 547-550.

[12] Kodiyath R., Malak S.T., et al. Assemblies of silver nanocubes for highly sensitive SERS chemical vapor detection. J. Mater. Chem. A, 2013, 1 (8), P. 2777-2788.

[13] Biggs K.B., Camden J.P., Anker J.N., Van Duyne R.P. Surface-enhanced Raman spectroscopy of benzenethiol adsorbed from the gas phase onto silver film over nanosphere surfaces: determination of the sticking probability and detection limit time. J. Phys. Chem. A, 2009, 113 (16), P. 4581-4586. 
[14] Zayak A.T., Hu Y.S., et al. Chemical Raman enhancement of organic adsorbates on metal surfaces. Phys. Rev. Lett., 2001, 106 (8), 083003(1-4).

[15] Stokes D.L., Pal A., Narayanan V.A., Vo-Dinh T. Evaluation of a chemical vapor dosimeter using polymer-coated SERS substrates. Anal. Chim. Acta, 1999, 399 (3), P. 265-274.

[16] Mueller M., Tebbe M., et al. Large-area organization of pNIPAM-coated nanostars as SERS platforms for polycyclic aromatic hydrocarbons sensing in gas phase. Langmuir, 2012, 28 (24), P. 9168-9173.

[17] Lin E.-C., Fang J., et al. Effective localized collection and identification of airborne species through electrodynamic precipitation and SERS-based detection. Nat. Commun., 2013, 4, 1636.

[18] Piorek B.D., Lee S.J., et al. Free-surface microfluidic control of surface-enhanced Raman spectroscopy for the optimized detection of airborne molecules. PNAS, 2007, 104 (48), P. 18898-18901.

[19] Rae S.I., Khan I. Surface enhanced Raman spectroscopy (SERS) sensors for gas analysis. Analyst, 2010,135 (6), P. $1365-1369$.

[20] Khaing Oo M.K., Chang C.-F., Sun Y., Fan X. Rapid, sensitive DNT vapor detection with UV-assisted photo-chemically synthesized gold nanoparticle SERS substrates. Analyst, 2011, 136 (13), P. 2811-2817.

[21] Kreno L.E., Greeneltch N.G., et al. SERS of molecules that do not adsorb on Ag surfaces: a metalorganic framework-based functionalization strategy. Analyst, 2014, 139 (16), P. 4073-4080.

[22] Chou A., Jaatinen E., et al. SERS substrate for detection of explosives. Nanoscale, 2012, 4 (23), P. 7419-7424.

[23] Khaing Oo M.K., Guo Y., et al. Ultrasensitive vapor detection with surface-enhanced Raman scattering-active gold nanoparticle immobilized flow-through multihole capillaries. Anal. Chem., 2012, 84 (7), P. 3376-3381.

[24] Wang J., Yang L., et al. Spectroscopic ultra-trace detection of nitroaromatic gas vapor on rationally designed two-dimensional nanoparticle cluster arrays. Anal. Chem., 2011, 83 (6), P. 2243-2249.

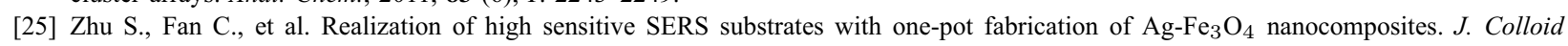
Interface Sci., 2015, 438, P. 116-121.

[26] Chong X., Kim K.J., et al. Plasmonic nanopatch array with integrated metal-organic framework for enhanced infrared absorption gas sensing. Nanotechnology, 2017, 28, 26LT01.

[27] Zhang Z., Yu W., et al. Ultrasensitive surface-enhanced Raman scattering sensor of gaseous aldehydes as biomarkers of lung cancer on dendritic ag nanocrystals. Anal. Chem., 2017, 89 (3), P. 1416-1420.

[28] Chen Y., Zhang Y., et al. Breath analysis based on surface-enhanced Raman scattering sensors distinguishes early and advanced gastric cancer patients from healthy persons. ACS Nano, 2016, 10 (9), P. 8169-8179.

[29] Wong C.L., Dinish U.S., Schmidt M.S., Olivo M. Non-labeling multiplex surface enhanced Raman scattering (SERS) detection of volatile organic compounds (VOCs). Anal. Chim. Acta, 2014, 844, P. 54-60.

[30] Gerardin C., Reboul J., Bonne M., Lebeau B. Ecodesign of ordered mesoporous silica materials. Chem. Soc. Rev., 2013, 42 (9), P. 42174255.

[31] Innocenzi P., Malfatti L. Mesoporous thin films: properties and applications. Chem. Soc. Rev., 2013 , 42 (9), P. 4198-4216.

[32] Fleishmann M., Hendra P.J., McQuillan A.J. Raman spectra of pyridine adsorbed at a silver electrode. Chem. Phys. Lett., 1974, 26 (2), P. $163-166$.

[33] Morris V.R., Bhatia S.C., Stelson A.W., Hall J.H. Matrix - isolation study of thermal decomposition of pyridine. Energy and Fuel, 1991, 5, P. 126-133. 